Journal of Oral Science, Vol. 59, No. 2, 201-206, 2017

Review

\title{
The oral microbiome and human health
}

\author{
Yoshihisa Yamashita1) and Toru Takeshita1,2) \\ 1)Section of Preventive and Public Health Dentistry, Division of Oral Health, Growth and Development, \\ Kyushu University Faculty of Dental Science, Fukuoka, Japan \\ 2)OBT Research Center, Kyushu University Faculty of Dental Science, Fukuoka, Japan
}

(Received December 13, 2016; Accepted January 23, 2017)

\begin{abstract}
In this brief review, we discuss our previous research on the relationship between the bacterial composition of salivary microbiota and periodontal disease. Analysis using a terminal restriction fragment length polymorphism method and an international comparison suggest that the predominance of the genera Prevotella and Veillonella in the salivary microbiota is attributable to periodontal disease conditions, and that the predominance of the genus Neisseria indicates healthy periodontal conditions. Furthermore, we recently used next-generation sequencing technology to perform a detailed largescale analysis of the salivary microbiota. An important finding of that study was that high bacterial richness in the salivary microbiota was significantly associated with poor oral health, as indicated by decayed teeth, periodontitis, and poor oral hygiene. Another important result was that relative abundance of predominant bacteria in saliva was significantly associated with oral health-related conditions. Of the two different cohabiting groups of bacteria found in the salivary microbiota, a greater relative abundance of group I bacteria, which include Prevotella and Veillonella species, was associated with poor oral health, high body mass index, and old age. These findings suggest that the salivary microbiota reflects oral and systemic conditions.
\end{abstract}

Correspondence to Dr. Yoshihisa Yamashita, Section of Preventive and Public Health Dentistry, Division of Oral Health, Growth and Development, Kyushu University Faculty of Dental Science, 3-1-1 Maidashi, Higashi-ku, Fukuoka 812-8582, Japan

Fax:+81-92-642-6354 E-mail: yoshi@dent.kyushu-u.ac.jp

doi.org/10.2334/josnusd.16-0856

DN/JST.JSTAGE/josnusd/16-0856
Keywords: oral microbiome; next generation sequencing; 16S rRNA; oral health; systemic health.

\section{Introduction}

The oral microbiome was first recognized by the Dutchman Antony van Leeuwenhoek, using a microscope of his own construction. In the late 1670s he reported to the British Royal Society that various forms of microbes were present in plaque found on tooth surfaces. This observation came at a time when bacteriology was not yet established, and he was immensely fascinated by the kineticism of the microbiome. His report described individual differences in the oral microbiome, and although he did not refer to it directly he realized that individual differences in the microbiome influenced the health of the oral cavity. Unfortunately, it would be 200 years before we developed a systematic theory that showed how the microbiome is related to disease in the oral cavity.

The American dentist W.D. Miller studied the association between oral microbes and oral diseases in a small laboratory in Berlin in the late 19th century. He was motivated by R. Koch, who, at about the same time, had been reporting cutting-edge results in studies of the association between microbes and infectious diseases. In his book, The Micro-organisms of the Human Mouth, Miller proposed the "chemicoparasitic theory", which held that the main cause of dental caries was acid metabolized from sugar in food by oral microbes. However, he failed to identify cariogenic bacteria and focused on acids as bacterial metabolites rather than on the bacteria that produced these metabolites. Until the mid-20th century, it is believed that his theory misled his successors, who categorized the Lactobacillus species as cario- 
genic bacteria, in accordance with Koch's hypothesis. However, was it correct to pursue a specific bacterium as the cause of oral diseases affected by a complex oral microbiome? Was it wrong for Miller to identify a cariogenic bacterium that would reflect the true cause of oral diseases? His chemicoparasitic theory had features in common with more recent microbiome disease concepts. In addition, descriptions of the association between oral microbes and systemic health in The Micro-organisms of the Human Mouth remind us of the recent concept of periodontal medicine and aspiration pneumonia, and reflect his important scientific insights.

Dental researchers regard the discoveries of mutans streptococci (1), in the middle of the 20th century, and the specific periodontal pathogens Aggregatibacter actinomycetemcomitans (2), Porphyromonas gingivalis, Treponema denticola, and Tannerella forsythia (3), thereafter, as elements of the classical infectious etiology proposed by Koch. However, mutans streptococci are not frequently detected in dentin lesion caries. In addition, $P$. gingivalis, the most promising pathogenic candidate for periodontitis, affected periodontal tissue destruction in specific pathogen-free mice but not to a significant extent in germ-free mice. P. gingivalis is thus now regarded as a keystone bacterium that determines the virulence of the overall oral microbiome in periodontitis rather than as a pathogen directly destructive to periodontal tissue (4). Although other candidate periodontal pathogens are frequently detected in periodontal lesions, causal relationships are difficult to demonstrate, and we cannot exclude the possibility that these bacterial species proliferate while adapting to the environment of deep periodontal pockets. The pathogenicity of mutans streptococci and specific pathogens remains controversial, and the classical infectious etiology of Koch cannot explain fully the cause of oral diseases, dental caries, and periodontitis.

More than 700 bacterial species inhabit the oral cavity and make up the oral microbiome (5-7), and we cannot ignore the existence of the oral microbiome when considering bacterial virulence. Although each species in the microbiome exhibits lower virulence as compared with mutans streptococci or the previously mentioned candidate periodontal pathogens, cell numbers may be higher for less virulent species than for species with greater virulence. Alternatively, the interaction among bacterial species in the oral microbiome may regulate expressions of virulence factors in species with greater virulence. Therefore, mere accumulation of a higher virulence species is insufficient to determine the virulence of the overall microbiome. This is the concept of virulence of the oral microbiome, and it warrants comprehensive analysis.

\section{What is the oral microbiome?}

The oral cavity is the initial part of the digestive tract and is followed by the esophagus, stomach, intestine, and colon. The composition of the microbiome is different in each part of this tract and depends on the unique environment of the digestive organ $(8,9)$. Among digestive organs, the oral cavity is unusual because of the presence of teeth and hard tissues. In addition, oral mucosa is composed of covering mucosa (oral floor, buccal region, labial region, soft palate), masticatory mucosa (gingival region and hard palate), and specialized mucosa (tongue dorsum). Although the entire oral mucosa is covered by a layer of squamous epithelium, each structure is distinctive. The covering mucosa is covered by non-keratinized epithelium, while the masticatory mucosa is covered by keratinized epithelium. The surface of specialized mucosa comprises several types of papilla and has a complex structure. Different niches, including tooth surfaces, form within the oral cavity and have varied microbiome compositions, due to the distinct environmental conditions at each site. The bacterial composition of plaque on tooth surfaces differs between the supragingival and subgingival regions (10). In addition, the extent of the difference in composition is positively correlated with periodontal pocket depth and periodontitis progression (11). Furthermore, the bacterial composition of saliva includes bacteria detached from various niches in the oral cavity and resembles that of the tongue coating $(8,9,12,13)$.

Alternatively, we need to consider temporal changes in the microbiome (14) when we examine individual specificity in microbiome composition. Temporal changes during supragingival plaque formation vary among individuals (15). Therefore, if a representative sample of plaque is required on one occasion, plaque maturity should be determined. Without information on plaque maturity, a sample of tooth surfaces is insufficient for analyzing the association between bacterial composition of plaque and health conditions. Specifically, when comparing bacterial composition of the microbiome, as a single variable, with a health condition, it is important to know two other sample variables: the sampling site and maturity. Because sampling the oral microbiome is easier than sampling other regions of the digestive tract, it is often the focus of analysis, both for dental researchers and microbiologists in other fields. However, if the above conditions are not considered, the analysis may yield inaccurate conclusions. We emphasize that, to understand the association between the oral microbiome and overall 
health, it is important to define the oral microbiome and recognize the complexity of the oral cavity.

\section{The salivary microbiome and its relation- ship with periodontal diseases}

When considering direct relationships between the microbiome and oral lesions (e.g., dental caries and periodontitis), the bacterial composition of dental plaque should be the focus because these lesions are directly exposed to dental plaque. Pathogens responsible for dental caries and periodontitis are believed to be located in supragingival and subgingival plaque, respectively. Removal of dental plaque is the fundamental measure for preventing oral disease. However, the oral microbiome may regulate the virulence of specific bacteria in dental plaque. Furthermore, accumulation of virulent bacteria should be considered for a bacterium with a large relative abundance, even if its individual virulence is quite low.

The microbiome in saliva is shed from various niches in the oral cavity and appears to be representative of the overall oral microbiome. We previously analyzed microbiome saliva from 200 subjects and compared the results with periodontal health conditions (16). The analysis was performed by using a terminal restriction fragment length polymorphism (T-RFLP) method (17) in which amplicons from the 16S rRNA gene were digested with a restriction enzyme. Fluorescence-labeled terminal fragments were then separated and detected by capillary electrophoresis. Although the T-RFLP method has been superseded by next-generation sequencing and rapidly improved molecular genetic methods, it was a powerful molecular genetic technique. In addition, the results obtained with our modified T-RFLP method (18) are roughly equivalent to those obtained by next-generation sequencing technology in our recent analyses. Therefore, we will discuss the results of our T-RFLP analysis of the association between the salivary microbiome and periodontitis.

Peak patterns from the saliva of the 200 subjects were obtained with the T-RFLP method. The data were then cluster-analyzed and classified into three clusters (I, II, and III) by using partitioning around medoids clustering. Principal component analysis of the similarity of peak patterns clearly showed that these three clusters were separate and were characteristically plotted on a twodimensional plane. Peaks characterizing cluster I were assigned to the genera Prevotella and Veillonella, those characterizing cluster II were assigned to the genus Streptococcus, and those characterizing cluster III were assigned to the genera Porphyromonas and Neisserial Haemophilus/Aggregatibacter. Samples representing each cluster were analyzed with the clone library method, and the results were consistent with those obtained by our improved T-RFLP method. This suggests that our T-RFLP method yielded reliable and accurate data on microbiome composition. Periodontal health condition was compared among these three clusters. On the basis of periodontal pocket depth and gingival bleeding, periodontal tissue condition was healthiest in cluster III and least healthy in cluster I. Pocket depth was similar in clusters I and II, but the number of bleeding sites was lower for cluster II than for cluster I.

These results have not been readily accepted by many dental researchers because the microbiome in saliva significantly differs from that of subgingival plaque, and subgingival tissue is not directly exposed to the microbiome of saliva. In fact, many reviewers of our report raised criticism about why we evaluated the microbiome of saliva but not that of subgingival plaque. Subsequently, we confirmed the association between the saliva microbiome and periodontal health in a comparison of Japanese and Korean populations (19). National surveys of dental health in Japan and Korea revealed that periodontal health was better in Korea, even though socioenvironmental factors related to periodontal health (e.g., oral hygiene behavior, smoking habit, number of dentists per capita, and coverage of dental therapy by public health insurance) were more favorable in Japan. We hypothesized that oral environment rather than the above socioenvironmental/socioeconomic factors was responsible for the observed differences in periodontal health conditions between Japanese and Koreans. The most promising candidate in the oral environment was the salivary microbiome, and we therefore compared the bacterial composition of the salivary microbiomes in the Japanese and Korean cohorts.

In that analysis, we selected orally healthy subjects from two cohort studies, because oral health conditions greatly affect the bacterial composition of the salivary microbiome. The two orally healthy populations from Japan and Korea exhibited different salivary microbiome patterns: Neisseria and Haemophilus were more prevalent among Koreans, while Prevotella and Veillonella were more prevalent among Japanese. Interestingly, this difference was identical to that observed between clusters III and I in the Japanese population described above. That is, the salivary microbiome of Koreans was most like cluster III while that of Japanese was most like cluster I, even though both populations were orally healthy. These results suggest that the salivary microbiome, which reflects the oral environment, indirectly affects periodontal health. However, differences in the 
Table 1 Predominant species-level operational taxonomic units (OTU) in the salivary microbiome of 2,343 adult inhabitants of Hisayama Town (20)

\begin{tabular}{lllc}
\hline OTU & Relative \\
No. & Bacterial species corresponding to the OTU & $\begin{array}{l}\text { Prevalence } \\
\text { abundance }(\%)\end{array}$ \\
\hline OTU3 & Neisseria flavescens $(610)$ & $9.3 \pm 9.2$ & 99.3 \\
OTU1 & Rothia mucilaginosa (681) & $8.8 \pm 7.2$ & 100 \\
OTU2 & Prevotella melaninogenica (469) & $8.7 \pm 6.0$ & 100 \\
OTU14 & Streptococcus mitis (677) & $4.3 \pm 3.1$ & 100 \\
OTU479 & Genus Streptococcus & $3.6 \pm 3.0$ & 100 \\
OTU12 & Streptococcus salivarius (755) & $3.3 \pm 3.7$ & 99.9 \\
OTU89 & Genus Actinomyces & $3.3 \pm 3.0$ & 99.9 \\
OTU7 & Granulicatella adiacens (534) & $3.0 \pm 1.9$ & 100 \\
OTU8 & Porphyromonas pasteri (279) & $2.5 \pm 3.1$ & 97.3 \\
OTU5 & Veillonella. atypica (524) & $2.3 \pm 1.6$ & 100 \\
\hline
\end{tabular}

${ }^{\mathrm{a}} \mathrm{Mean} \pm \mathrm{SD}$; ${ }^{\mathrm{b}}$ Oral taxon IDs in the Human Oral Microbiome Database (7) are shown in parentheses.

microbiome are not derived from an "all-or-nothing" relationship among specific bacteria. The clustering of the microbiome is believed to be due to differences in the relative abundance of bacteria common to both clusters. This is reflected in the difference in the microbiomes of the Japanese and Korean cohorts. Thus, a healthy microbiome is achieved by comprehensive overall regulation of bacterial species, not by elimination of specific virulent bacteria.

The mechanisms by which differences in the salivary microbiome affect periodontal health are not well understood. However, if our hypothesis is correct, elimination of microbiota on tooth surfaces (e.g., subgingival and supragingival plaque) is insufficient to control periodontal health. Instead, periodontal therapy should direct the salivary microbiome toward healthy patterns. There are no effective measures to control the microbiome in saliva; however, controlling tongue coating may be a promising periodontal therapy, given the similarity of the salivary microbiome and tongue coating. Nevertheless, international comparisons of socioeconomic status might help identify environmental factors that affect the difference in salivary microbiomes and provide insights useful for programs in periodontal disease prevention, such as restriction of sucrose consumption and fluoride application for dental caries prevention.

\section{Large-scale analysis of the Japanese salivary microbiota}

Recently, we performed a large-scale populationbased study of the salivary microbiome to confirm the above-mentioned evidence on oral health conditions and examine the effects on systemic conditions (20). We collected saliva from residents of Hisayama Town, Japan, and used the 16S rRNA gene and next-generation sequencing technology (Ion PGM) to determine micro-

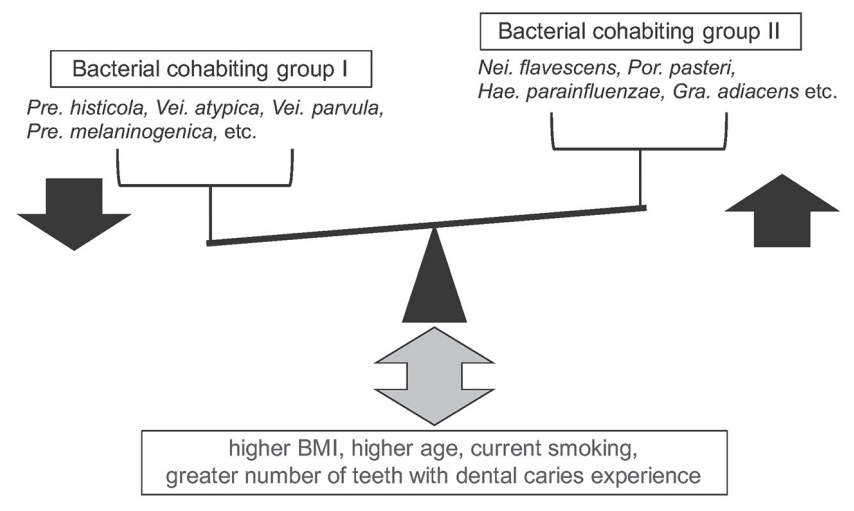

Fig. 1 The relationship between the relative abundance of common predominant bacteria in saliva and health conditions (20).

biota composition in 2,343 adults aged 40 years or older. An important finding was that high bacterial richness in a salivary microbiome was significantly associated with poor oral health, including the presence of decayed teeth, periodontitis, and poor oral hygiene. This result is understandable and was expected because pathogenic plaque bacteria in diseased sites additionally shed into saliva. In fact, a variety of well-known pathobionts, including $S$. mutans and $P$. gingivalis, were more frequently identified in the salivary microbiota of individuals with poor oral health. A novel finding was that relative abundances of predominant bacteria in saliva were significantly associated with oral health-related conditions. Most organisms in the salivary microbiota are present in almost all individuals, including Streptococcus, Neisseria, Rothia, Prevotella, Actinomyces, Granulicatella, Porphyromonas, Haemophilus, and Porphyromonas species (Table 1). Data on bacterial composition for the 2,343 participants suggest that these predominant organisms comprise two different cohabiting groups of bacteria: 
one mainly composed of Prevotella histicola, Prevotella melaninogenica, Veillonella parvula, Veillonella atypica, Streptococcus salivarius, and Streptococcus parasanguinis (bacterial cohabiting group I), the other primarily assembled from Neisseria flavescens, Haemophilus parainfluenzae, Porphyromonas pasteri, Gemella sanguinis, and Granulicatella adiacens (bacterial cohabiting group II). The clustering approach classified the composition of the salivary microbiota into two types (community types I and II), which had different relative abundances of these bacterial cohabiting groups. A larger relative abundance of bacterial cohabiting group I was associated with poorer health conditions (Fig. 1). This result was consistent with our previous findings from the T-RFLP approach and added detailed information on the salivary microbiota. Furthermore, a significant relationship was observed between their predominance and systemic conditions, such as high body mass index and old age, even after controlling for confounding effects in multivariate analysis. These results suggest that the salivary microbiota reflects both the oral and systemic condition of an individual. The overall composition of the oral microbiome was revealed by a comprehensive molecular approach based on bacterial DNA. However, the role of each organism in the oral microbiome, especially bacteria without an apparent virulence factor, is not fully understood. The relationship between overall health and the balance of the cohabiting groups of common predominant bacteria is a topic that requires further investigation.

In this article, we discussed the relationship of the salivary microbiota with periodontal health and systemic conditions. The recent rapid development of molecular genetics in high-throughput DNA sequencing enables us to grasp important aspects of the oral microbiota and its association with oral and systemic health. These studies may lead to promising dental treatments and interventions to manage the bacterial composition of the oral microbiome.

\section{Acknowledgments}

This study was supported in part by Grant-in Aid for Scientific Research from Japan Society for the Promotion of Science (16H02692, 16H05850, 15K15774, 16H05557). We are very grateful to Professor Noriaki Koshikawa for encouraging our research and for his contribution to the development of orofacial neuroscience.

\section{Conflict of interest}

The authors declare that they have no competing interests.

\section{References}

1. Kuramitsu HK (1993) Virulence factors of mutans streptococci: role of molecular genetics. Crit Rev Oral Biol Med 4, 159-176.

2. Irving JT, Newman MG, Socransky SS, Heely JD (1975) Histological changes in experimental periodontal disease in rats mono-infected with a gram-negative organism. Arch Oral Biol 20, 219-220.

3. Socransky SS, Haffajee AD, Cugini MA, Smith C, Kent RL Jr (1998) Microbial complexes in subgingival plaque. J Clin Periodontol 25, 134-144.

4. Hajishengallis G, Liang S, Payne MA, Hashim A, Jotwani R, Eskan MA et al. (2011) Low-abundance biofilm species orchestrates inflammatory periodontal disease through the commensal microbiota and complement. Cell Host Microbe 10, 497-506.

5. Aas JA, Paster BJ, Stokes LN, Olsen I, Dewhirst FE (2005) Defining the normal bacterial flora of the oral cavity. J Clin Microbiol 43, 5721-5732.

6. Dewhirst FE, Chen T, Izard J, Paster BJ, Tanner AC, Yu WH et al. (2010) The human oral microbiome. J Bacteriol 192, 5002-5017.

7. Chen T, Yu WH, Izard J, Baranova OV, Lakshmanan A, Dewhirst FE (2010) The Human Oral Microbiome Database: a web accessible resource for investigating oral microbe taxonomic and genomic information. Database (Oxford), doi:10.1093/database/baq013.

8. Segata N, Haake SK, Mannon P, Lemon KP, Waldron L, Gevers D et al. (2012) Composition of the adult digestive tract bacterial microbiome based on seven mouth surfaces, tonsils, throat and stool samples. Genome Biol 13, R42.

9. Zhou Y, Gao H, Mihindukulasuriya KA, La Rosa PS, Wylie KM, Vishnivetskaya $T$ et al. (2013) Biogeography of the ecosystems of the healthy human body. Genome Biol 14, R1.

10. Ximenez-Fyvie LA, Haffajee AD, Socransky SS (2000) Comparison of the microbiota of supra- and subgingival plaque in health and periodontitis. J Clin Periodontol 27, 648-657.

11. Griffen AL, Beall CJ, Campbell JH, Firestone ND, Kumar PS, Yang ZK et al. (2012) Distinct and complex bacterial profiles in human periodontitis and health revealed by $16 \mathrm{~S}$ pyrosequencing. ISME J 6, 1176-1185.

12. Mager DL, Ximenez-Fyvie LA, Haffajee AD, Socransky SS (2003) Distribution of selected bacterial species on intraoral surfaces. J Clin Periodontol 30, 644-654.

13. Zaura E, Keijser BJ, Huse SM, Crielaard W (2009) Defining the healthy "core microbiome" of oral microbial communities. BMC Microbiol 9, 259.

14. Ritz HL (1967) Microbial population shifts in developing human dental plaque. Arch Oral Biol 12, 1561-1568.

15. Takeshita T, Yasui M, Shibata Y, Furuta M, Saeki Y, Eshima $\mathrm{N}$ et al. (2015) Dental plaque development on a hydroxyapatite disk in young adults observed by using a barcoded pyrosequencing approach. Sci Rep 5, 8136.

16. Takeshita T, Nakano Y, Kumagai T, Yasui M, Kamio N, 
Shibata Y et al. (2009) The ecological proportion of indigenous bacterial populations in saliva is correlated with oral health status. ISME J 3, 65-78.

17. Liu WT, Marsh TL, Cheng H, Forney LJ (1997) Characterization of microbial diversity by determining terminal restriction fragment length polymorphisms of genes encoding 16S rRNA. Appl Environ Microbiol 63, 4516-4522.

18. Takeshita T, Nakano Y, Yamashita Y (2007) Improved accuracy in terminal restriction fragment length polymorphism phylogenetic analysis using a novel internal size standard definition. Oral Microbiol Immunol 22, 419-428.

19. Takeshita T, Matsuo K, Furuta M, Shibata Y, Fukami K, Shimazaki Y et al. (2014) Distinct composition of the oral indigenous microbiota in South Korean and Japanese adults. Sci Rep 4, 6990.

20. Takeshita T, Kageyama S, Furuta M, Tsuboi H, Takeuchi K, Shibata Y et al. (2016) Bacterial diversity in saliva and oral health-related conditions: the Hisayama Study. Sci Rep 6, 22164. 